\title{
FEAR DOES NOT CORRESPOND TO THE HIGHER COSTS OF RAPE AMONG MARRIED WOMEN
}

\author{
JefFrey Keith SNyder, Ph.D. ${ }^{* 1}$, DANiel M.T. Fessler, Ph.D. ${ }^{2}$ \\ ${ }^{1,2}$ Los Angels, California UNITED STATES
}

\begin{abstract}
THORNHILL and THORNHILl posited that sexual assault inflicts greater fitness costs on women in committed long-term heterosexual relationships than on women not in such relationships because the former face the added risk of decreased investment by their partners. In a series of papers (1990a, 1990b, 1990c, and 1991), THORNHILL and THORNHILL reported support for what we term the Relationship Status Hypothesis (RSH) using data on the psychological sequelae of rape. Here, we reexamine the RSH in light of THORNHILL and THORNHILL's original findings and the relevant literature. Identifying limitations of the original work and finding little support for the RSH in other published work, we then test the RSH in two studies using prospective fear of rape as a dependent measure; again, we find no support for the hypothesis. We conclude that, although marital discord following rape is an important issue warranting further study, the Relationship Status Hypothesis has limited empirical support at present.
\end{abstract}

Keywords: sexual coercion, rape, rape avoidance, evolved psychological mechanisms

\section{INTRODUCTION}

Some two decades ago, THORNHILL and THORNHILL (henceforth T \& T) published a series of four articles (1990a; 1990b; 1990c; 1991) addressing women's psychological pain following rape from an evolutionary perspective. $T \& \mathrm{~T}$ posited that psychological pain, being a subjective proxy for fitness decrements suffered, is a proximate mechanism motivating a functional change in behavior that decreases the probability of suffering similar costs in the future. Building on this premise, T \& T's central claim was that variation across women in the potential fitness costs of rape corresponds with variation in the psychological pain experienced following rape. T \& T (1990a, 1990c; see also THORNHILL 1996) hypothesized that, compared with unmarried women, married women face an added cost as a result of sexual assault, namely reduced investment on the part of their male romantic partner, as a woman's partner might recalibrate his willingness to provide benefits to her and her children either due to an interpretation of the assault as resulting from extra-pair solicitation by the woman (i.e., indexing heightened risk of infidelity), or due simply to the drain on male parental investment posed by the rapist's offspring should conception have occurred. A core tenet in T \& T's framework was thus that, following

* Address for correspondence: Jeffrey KeIth Snyder, Ph.D. ${ }^{1}$ Department of Anthropology UCLA, Los Angels, California UNITED STATES, E-mail: jksnyder@ucla.edu DANIEL M.T. Fessler, Ph.D. ${ }^{2}$ UCLA, Los Angels, California UNITED STATES, E-mail: dfessler@anthro.ucla.edu 
rape, women of reproductive age who are married would experience more psychological pain than unmarried women of similar age; henceforth we refer to this notion as the Relationship Status Hypothesis, or RSH.

Paralleling their conclusions regarding the effects of marital status on psychological pain following rape, $\mathrm{T} \& \mathrm{~T}$ also asserted that because rape potentially imposes greater costs on women of reproductive age than on those who are pre- or post-reproductive, corresponding age-related differences occur in psychological pain (1990a). While authors such as FREESE (2000) and Koss (2000) have extensively criticized the latter claim on empirical grounds, T \& T's important conclusion that relationship status moderates the severity of psychological pain associated with rape has yet to be critically examined.

The RSH is cogent and thus worthy of investigation. However, rather than seeking to further test this hypothesis, much subsequent work has instead taken it as proven. Viewed historically, given the frequency with which T \& T's work has been cited, this trajectory is likely the product of iterated citation chains involving successive diminution in the reporting of the details of the original studies, with the nuances and limitations of the original conclusions being lost along the way. Importantly, the impact of such a process ramifies beyond the scientific community, as, given its social significance, approaches such as this have implications for jurisprudence and public policy (e.g., JONES 1999).

Given both its cogency and its social importance, the RSH merits further examination. T \& T's pioneering work is one of the few instances in which archival rape data has been used to directly test evolutionary hypotheses (see also MORGAN, 1981; ROGEL 1976; WILSON and MESNICK 1997). In light of the difficulty of conducting such research, here we adopt a multi-pronged strategy in evaluating the $\mathrm{RSH}$. First, we reexamine the original findings in detail. Second, we compare these findings with results published elsewhere in the literature. Third, we employ new survey data to provide indirect tests of the RSH.

\section{REEXAMINING THE RELATIONSHIP STATUS HYPOTHESIS}

\subsection{The Original Studies}

In the first of their four articles, T \& T (1990a) sought to establish the predicted effects of age and relationship status on psychological pain following rape. As is true in the three subsequent papers as well, T \& T (1990a) employed questionnaire data from a longitudinal study of 790 sexual assault victims. Victims were recruited for participation from a Philadelphia, PA hospital from 1973 to 1975. In order to study the victims' psychological adjustment following rape, they were interviewed quarterly over a period of one year (MCCAHILL, MEYER and FISCHMAN 1979). However, only the data from the initial interview, conducted within five days of the rape, were analyzed by T \& T. The data included demographic information, the specifics of the assault, and thirteen variables intended to measure the victim's psychological 
adjustment immediately following the rape (MCCAHILL, MEYER and FISCHMAN, 1979; THORNHILL and THORNHILL 1990a).

Because of likely connections between age and marital status, so as to provide a context for understanding T \& T's exploration of the RSH, we begin with a description of their investigations of the effects of the victim's age on psychological pain. T \& T examined age effects by partitioning the sample into three groups: prereproductive (ages $0-11$ ), reproductive (ages 12-44), and post-reproductive (ages 45-88). The groups were then compared on each measure of psychological trauma - such as a change in eating patterns, change in social activities, and change in fear of unknown men - with both Chi-square analysis and Kruskal-Wallis analysis. First, Chi-square analysis was used to determine if there were significant differences between the non-reproductive-aged women and reproductive-aged women. As predicted, eight of the thirteen variables of psychological trauma showed a significant change in magnitude among victims of rape within five days of the assault. However, statistical corrections for repeated tests (such as BONFERRONI or FISHER'S LSD), as appropriate in cases in which multiple tests are conducted to test for a single hypothesis (SHAFFER 1995), were not employed. Follow-up analysis with the three age categories (pre-reproductive, reproductive and post-reproductive) with Kruskal-Wallis nonparametric analysis of variance suggested that "in general pre-reproductive-aged girls are far less likely to [be] psychologically traumatized by rape and reproductive-aged women are far more likely to be so than expected under the null hypothesis" (1990a, pp. 164-166). However, the strength of these conclusions is diminished when the appropriate Bonferroni correction is applied, as only six out of thirteen variables then remain significant.

To explore the effects of marital status, $\mathrm{T} \& \mathrm{~T}$ first truncated the sample to exclude pre-reproductive victims, leaving a sample composed of women age 12-88. T $\& \mathrm{~T}$ then reduced the five marital categories (unmarried, married, widowed, separated, and divorced) employed in the original data set to two categories (married and unmarried), with the married category including separated women on the premise that separated women were still being at least partly provisioned by mates, and hence face similar costs of a reduction in investment to those faced by married women. The unmarried category included single, divorced and widowed victims of sexual assault. The remaining sample was then examined using thirteen Chi-square analyses, one for each of the psychological trauma variables. These analyses revealed a significant difference in married women's level of trauma on six of thirteen variables - a change in eating patterns, a change in sleeping patterns, a change in frequency of nightmares, fear of being on the street alone, fear of strange men, and fear of being home alone (note the importance of fear, a topic to which we will return later). T \& $\mathrm{T}$ also pointed to non-significant trends in indicators of worsened relationships with men, including their partners, within five days of the assault. Additionally, noting that married women were more likely to intend to seek psychological assistance following rape, $\mathrm{T} \& \mathrm{~T}$ suggested that this similarly indicated that married women suffered more negative psychological impact than unmarried 
women. Likewise, lending additional support to the RSH, married women were also significantly more likely to report that they felt that their futures had been "profoundly affected by the rape" (1990a, p. 171). However, the significance of frequency of nightmares and fear of unknown men do not hold up to Bonferroni correction. Frequency of nightmares drops from $p=.020$ to $p=.26$, and fear of unknown men drops from $p=.008$ to $p=.104$. Again, fewer than half of the variables remain significant after the appropriate correction is applied. Likewise, the nonsignificant trends described by T \& T are not noteworthy after Bonferroni correction.

T \& T (1990a) recognized that age and marital status are likely to be confounded. Because most reproductive-aged women in the sample were married, to ensure that marital status was not driving the age effect, the age effect - that reproductive-aged women experience greater psychological pain than women of other age groups - was retested using only the "unmarried" subsample. This analysis yielded five significant effects out of thirteen tests; only four of these remain significant after Bonferroni correction (change in sleep pattern drops from $p=.036$ to $p=.468$ ). Importantly, however, despite recognizing that age and marital status were confounded, $\mathrm{T} \& \mathrm{~T}$ did not perform the necessary converse retests - although it is likely that women in the married subsample were older than those in the unmarried subsample, no analyses were performed to ensure that the effects of age were not driving the difference between the married and unmarried subsamples.

To summarize the above, T \& T's original studies do not provide clear support for the RSH due to the manner in which the sample was treated, the logic of the analyses, the exclusive use of non-parametric statistical tests, and the omission of corrections for multiple statistical tests (for additional concerns, see BROWNMILLER, 2000; Coyne 2000; COYNe and BERRY 200; FreEse 2000; and Koss 2000). ${ }^{1}$ SHARING FREESE'S (2000) sentiments that the original data should be reanalyzed using superior methods, we attempted to obtain the original archival files from the Peters Institute in Philadelphia. However, not surprisingly given the many decades that have passed, it seems that the original data no longer exist.

Given that the original data are not available, ideally, we would be able to test the predictions generated by the RSH using comparable data from other rape victims. However, such data are very difficult to obtain. Instead, we examine other published research concerning the aftermath of sexual assault as an alternative avenue for evaluating the RSH.

\subsection{The Relationship Status Hypothesis Reexamined: Review of Literature}

Prior to 1990, perhaps the most influential work on the effect of relationship status and women's adjustment following rape was that of MCCAHILL, MEYER, and FISCHMAN (1979). Their work evaluated the same data set used by T \& T, albeit with an important difference: MCCAHILL et al.'s data set included narratives and follow-up interviews assessing women's psychological adjustment approximately 
one year post-rape. MCCAHILL et al. report that, “A married woman is nearly twice as likely to experience worsened relationships with her husband than is an unmarried woman with her boyfriend (45.5\% versus 23.5\%)," (p. 48). This observation can be interpreted as consonant with T \& T's (1990a) analysis of psychological pain in the immediate aftermath of rape, as such pain could conceivably foreshadow subsequent elevated potential for marital discord. Qualitative interpretation of victims' narratives led MCCAHILL et al. to suggest three reasons why sexual assault may increase marital stress: the husband may blame the victim in a general way for simply being the source of their marital stress; the husband may be uncertain as to how best to console his wife as the victim; and, consonant with the RSH, the husband may accuse the wife of being complicit in the rape, or may blame the victimized wife for not offering enough resistance to the rape (pp. 47-48). In regard to the latter, MCCAHILL et al. state "Victims who bear no overt physical injuries are most likely to be confronted with this sort of accusation,” (p. 47). While consistent with the premises of the RSH, to the extent that long-term outcomes are indeed predicted by sequelae in the short term, this observation is nevertheless not in keeping with $\mathrm{T}$ \& T's results, as, in a separate analysis (1990c), they failed to find markedly decreased psychological pain among victims who were physically beaten in the course of the rape (see also Koss 2000 for similar null results). Lastly, according to MCCAHILL et al., an additional source of marital discord was a husband's self-blame for a failure to protect his spouse, sometimes followed by blame-shifting, a factor that is outside of the theoretical frame of the RSH, and could therefore have spuriously contributed to patterns that appear to support it.

Using a different sample, KILPATRICK, VERONEN, and BEST (1985) were not able to validate MCCAHILL, MEYER and FISCHMAN's (1979) observations. Kilpatrick et al. recruited 125 women aged 16 or older who were victims of coerced completed oral, anal, and/or vaginal intercourse. Demographic information was obtained (age, race, marital status, number of children, living arrangements, occupational status, and religious preference), and participants were asked to complete The Life Events Inventory, The Previous History Inventory, Rape Assault Characteristics Checklist, Profile of Mood States Scale, Self-report Inventory, The Derogatis Symptom Check List 90-R, The State-Trait Anxiety Inventory, and The VernonKirkpatrick Modified Fear Survey. Participants were initially interviewed between 6 and 21 days post-rape, and again at three months post-rape. Employing the above measures of stress and functioning, KILPATRICK et al. found no difference in psychological functioning and adjustment between married and unmarried victims. Similarly, RESICK, CALHOUN, ATKESON, and ELLIS (1981) conducted a year-long longitudinal study of 93 rape victims ranging in age from 15-71. Participants were interviewed initially within two weeks of the rape and afterwards at one month, two months, four months, eight months, and twelve months. Participants responded to the Social Adjustment Scale-Self Report, which includes measures of marital adjustment (level of intimacy, frequency of arguments, and degree of sexual satisfaction). RESICK et al. found no impairment of marital relations. However, one limita- 
tion in this study is that the marital assessment was aggregated with overall family assessment, apparently because there were too few married women in the study to perform factorial statistical analyses on the sub-sample of women co-habiting with romantic partners (see also RESICK 1993 for a comprehensive review of post-rape trauma up to that date).

Recently, McKibBin, SHACKelford, Miner, BATES, and LidDle (2011) reported results consistent with the RSH. Extrapolating from the premise that, due to the risk of reduced investment or abandonment, married women face an extra cost of rape, McKIBBIN et al. predicted that women in relationships will engage in more rape-avoidance behaviors. Using the Rape Avoidance Scale (RAI; McKIBBIN, SHACKELFORD, Goetz, BATES, and STARRATT 2009), MCKIBBin et al. found support for this prediction in a U.S. university sample. Specifically, women in heterosexual relationships scored higher on the Awareness of Surroundings/Defensive Preparedness and Avoid Appearing Sexually Receptive subscales of the RAI. On the face of it, McKiBBIN et al.'s results appear to provide indirect support for the RSH. However, as we have argued elsewhere (SNYDER \& FESSLER 2013), the RAI may be an inappropriate measure of married women's rape-avoidance behaviors, as many of the RAI items describe behaviors (such as "Avoid blind dates;" and "Avoid 'making out' with a man I have just met") in which married women are unlikely to engage at baseline. When ten such items with poor face validity are removed, the RAI no longer yields significant results for women in relationships (SNYDER and FESSLER 2013). Lastly, although the RAI, consisting of four subscales, has high inter-item reliability, being a relatively new measure, there are no reports to date on its construct validity. Seen in this light, MCKIBBIN et al.'s (2011) findings lend less support to the RSH than is apparent at first blush.

To summarize the above evaluation, based only on materials available to date, we suggest the following: First, nontrivial limitations hobble the analytic methods undergirding $\mathrm{T} \& \mathrm{~T}$ 's conclusion that married women experience more psychological pain as a result of rape, and T \& T's own subsequent work raises the possibility that said findings derive from confounding factors. Correspondingly, subsequent investigations have not supported this claim. In addition, to our knowledge, only one study has reported evidence consistent with this claim - that is, the work of MCCAHILL, MEYER and FISCHMAN (1979). Although MCCAHILL et al.'s qualitative interpretation of narrative material - drawn from the same sample as that which $\mathrm{T}$ \& T employed - is consistent with the RSH, MCCAHILL et al. also offer competing explanations, positing that the victim's male partner's perceived shortcomings as a protector may be a source of marital discord. In light of the evolutionary importance of the male protector (a.k.a. bodyguard) hypothesis (ELLIS 1992; SNYDER, FESSLER, TIOKHIN, FREDERICK, LEE, NAVARRETE 2011; WILSON and MESNICK 1997), below we will test this alternative explanation. Second, with the possible exception of MCCAHILL et al.'s qualitative observation that accusations of infidelity - a potential source of psychological pain that is consonant with the RSH - are more commonly directed at rape victims who were not physically injured, there is little evidence that 
women who have been beaten or have sustained physical injuries in the course of a sexual assault experience less psychological pain than other victims of rape. T \& T's own findings are null in this regard, and no work before or since supports this claim.

To summarize the above, underscoring the lack of conclusive support for the RSH in T \& T's original investigations thereof, other published work on the psychosocial aftermath of sexual assault largely fails to provide evidence consistent with the RSH. Given both its cogency and its influence on contemporary evolutionary work on rape, it is therefore important to subject the RSH to additional testing. We do so below by exploring prospective attitudes toward the risk of sexual coercion among women in the general population. ${ }^{2}$

\section{THE RELATIONSHIP STATUS HYPOTHESIS AND FEAR OF RAPE}

A core tenet of the RSH is that psychological pain, serving as a proxy for fitness costs, shapes behavior in an adaptive manner, where the degree of pain corresponds with the fitness value of taking steps to avoid repetitions of the pain-inducing events in the future. This is a highly plausible position - indeed, such an account is doubtless the ultimate explanation of pain in general. Against this backdrop, we believe that fear of rape can speak to the issue of the cost of rape to women. If it is the case that rape is more costly to married women than to unmarried women because rape increases the risk of diminished male investment, and if it is the case that taking precautions to reduce the risk of rape entails costs (in the form of time, attention, energy, and lost opportunities), then it should also be the case that married women are more likely to avoid circumstances associated with an elevated risk of sexual assault, i.e., married women should be more willing to pay the costs of precautions in order to avoid the greater costs that rape would impose on them (McKibBin, SHACKELFORD, MinER, BATES, and LidDLE 2011). The experience of fear or a fearful attitude toward sexual assault is plausibly the proximate motivator of such behaviors, as pain and fearfulness are necessarily functionally linked, since it is the fearful anticipation of pain that allows pain to prospectively shape behavior. We thus reason that women's fear of rape should track the costs of rape such that, the higher the cost of rape, the more likely a woman is to fear rape. This logic links T \& T's concept of psychological pain with the fear of rape construct in a manner that allows us to test the RSH beyond the narrow strictures of difficult-to-access samples composed of rape victims.

Following directly from the RSH, we predicted (i) that pair-bonded women's fear of rape would be greater than women who are not in committed long-term relationships. By extension, since the costs of post-rape reductions in a male partner's investment would be higher for a woman with offspring, we also predicted that (ii) pair-bonded women with offspring would express more fear of rape than both pairbonded women without offspring and single women. 
Before going forward, it is important to recognize that both of the above predictions are potentially subject to a confounding factor. MESNICK (1997) advanced the bodyguard hypothesis, suggesting that one functional benefit to females of forming heterosexual pair-bonds with males is reduced sexual harassment by other males (see also ELLIS, 1992; SNYDER, FESSLER, TIOKHIN, FrEDERICK, LEE, and NAVARRETE 2011). WILSON and MESNICK (1997) tested the bodyguard hypothesis among Canadian women. Analysis of a sample of 12,252 cases from Canadian national crime reports indicated that married women were less often victimized by lethal and nonlethal sexual assaults. If married women are less vulnerable to sexual assault, then they may experience less fear of sexual assault even though, were it to occur, rape would be more costly to them than to unmarried women - in effect, with regard to fear of rape, the higher cost of rape to married women may be canceled out by the lower probability of its occurrence. However, an alternative avenue of investigation allows us to address this problem. For inclusive fitness reasons, men living with their adult female kin should seek to protect the latter from sexual assault in a manner similar to that of husbands. However, unlike women residing with spouses, women residing with male kin do not face the potential cost of abandonment as a result of sexual assault. Given that single women living with male kin may enjoy similar protection from sexual assault compared to pair-bonded women, yet face fewer costs should they be so victimized, we therefore predicted that (iii) single women living with male kin would be less fearful of sexual assault than pairbonded women.

Finally, recall that $\mathrm{T} \& \mathrm{~T}$ predicted that relationship status and reproductive status should exercise independent effects on psychological pain following rape, and hence, by extension per our reasoning, on fear of rape. Recall also that a problem for T \& T's work in this regard is the asymmetry in their efforts to separate the effects of age and marital status, as they analyzed the former while controlling for the latter (by removing married women), but did not analyze the latter while controlling for the former. Finding an effect of age among single women does not reveal whether or not the married women who were removed from the sample were, on average, older than the unmarried women in the sample. If the latter applies, then age could have been solely responsible for the marital status effect. It is therefore important to revisit possible interactions between marital status and age. If $\mathrm{T} \& \mathrm{~T}$ were correct that the effects of age and marital status are independent, then it should be true that (iv) the effects of relationship status on fear of rape will be independent of age - that is, age will not exclusively mediate fear.

\section{STUDY 1}

\subsection{Details of Sample}

Data from a previous study (SNYDER, FESSLER, TIOKHIN, FREDERICK, LEE, NAVARRETE 2011) allowed for a preliminary exploration of these predictions. Em- 
ploying an Internet-based protocol and soliciting participants using postings on the Volunteers section of Craigslist.org, we had recruited 702 women age 18 and over from 38 large- and mid-sized cities in 30 U.S. states. Only women over the age of 18 were recruited. Homosexual women were excluded from the analyses that follow because homosexual women are less likely to encounter the risk of date rape, a common form of sexual assault. In addition, multiple responses from the same IP address were discarded. Due to the length of the survey, attrition in this study was high. The resulting sample was composed of 473 women ranging in age from 18 to 61 ( $\mathrm{M}=27.49, \mathrm{SD}=8.75)$; 75.5\% identified their ethnicity as White. The sample was well educated, with $93.3 \%$ reporting some higher education.

\subsection{Methods}

We asked participants to report household composition. They were provided with a checklist of potential forms of relationship to any persons in their household (for example, alone, roommate, mother, spouse, daughter \#1, daughter \#2, etc.). Women were classified as pair-bonded if they indicated that they were cohabitating with their romantic partner. Cohabitation is a better variable to use for this set of predictions than mere relationship status, as male investment is likely higher in the context of cohabitation, and hence the cost of abandonment to women in this context is likely higher. Women were classified as living with male kin if they reported living with their father, one or more adult brothers, or both. Fear of rape was measured with a modified version of the British Fear of Local Crime Survey Crime Scale (The Crime Reduction Centre, 2000). Participants identified their level of concern on a 1-4 scale from "not at all worried" to "very worried" with regard to being the victim of sexual assault, among other crimes.

\subsection{Results}

We tested the prediction that there would be a significant difference between women cohabitating with a romantic partner and women not cohabitating in fear of rape using an Independent Sample T-test. There was no significant difference between the former $(\mathrm{M}=2.31, \mathrm{SD}=0.94, n=162)$ and the latter $(\mathrm{M}=2.41$, $\mathrm{SD}=0.91, n=276)$ in this regard $(p=.86)$.

We also used an Independent Sample T-test to examine the prediction that there would be a significant difference between pair-bonded women with offspring, for whom the costs of abandonment should be highest, and single women, for whom no such costs exist - in short, by comparing women occupying the poles of the spectrum of theorized costs, we gave maximal opportunity for the RSH's costof-abandonment thesis to be supported. Nevertheless, analysis revealed no significant difference between pair-bonded women with offspring $(\mathrm{M}=2.37, \mathrm{SD}=0.95$, $n=114)$ and single women $(\mathrm{M}=2.41, \mathrm{SD}=0.90, n=276)(p=.54)$. 
The prediction that women living with male kin would be less fearful of sexual assault than women cohabitating with a heterosexual romantic partner was tested with a one-way ANOVA. We tested the dependent measure according to a threelevel variable (living with neither male kin nor a romantic partner, living with romantic partner only, and living with male kin only). No significant difference was found between single women not living with $\operatorname{kin}(\mathrm{M}=2.32$, $\mathrm{SD}=0.92, n=256)$, single women living with male kin $(2.50, \mathrm{SD}=0-90, n=94)$, and pair-bonded women living only with their romantic partners $(\mathrm{M}=2.40, \mathrm{SD}=0.95, n=88)$ $(p=.28)$.

We also computed post-hoc tests with Bonferroni correction and found that all individual mean comparisons between groups (living with neither male kin nor romantic partner, living with romantic partner only, and living with male kin only) were nonsignificant ( $p$ 's $>.34$ ). Lastly, having found no effect of relationship status/cohabitation on fear of sexual assault, it was not possible to test prediction (iv), the notion that age may be a confounding factor in any positive correlations between the aforementioned.

\subsection{Discussion}

None of the predictions derived from the RSH were supported. However, caution is always in order when interpreting null results. First, with regard to comparisons between cohabiting women and single women living with male kin, it is possible that the social dynamics of contemporary life in the U.S. are such that male kin do not provide the same level of protection from sexual assault as do male partners. If single women are not typically accompanied by male kin when engaging in activities, such as attending social events, that are perceived to pose a risk of sexual assault, then, contrary to our prediction, coresidence, with male kin may not reduce fear of rape in such women relative to those who cohabit with their partners. Accordingly, our failure to find a difference between these two categories may not be revealing. That said, the same limitations do not plague the straightforward comparison between cohabiting women and single women, yet this too failed to reveal a difference in fear of rape.

Second, more broadly, in addition to the possibility of sampling error, Study 1 may have produced null results due to limitations of our dependent measure, as we measured fear of rape using only a single self-report item. While this single item is straightforward, nevertheless, there are no reports of its stand-alone validity. Finally, the use of a 1-4 scale may encourage moderate responses, as the anchors of the scale, "Not at all worried" and "Very worried" may be perceived by participants as extreme responses. Additional data, collected in the course of a separate project, allowed us to address both of these possibilities through further tests of predictions (i) and (ii), employing a different sample and a more valid and reliable measure of fear of rape. 


\section{STUDY 2}

\subsection{Details of Sample}

Employing an Internet-based protocol and soliciting participants using postings on the Volunteers section of Craigslist.org and two web sites that serve to link participants with online psychology studies (socialpsychology.org and psych.hanover.edu), we recruited 333 women from 106 regions across the U.S. Attrition in this study too was high, again due to the length of the survey. Inclusion criteria were the same as in Study 1, and multiple responses from the same IP address were again discarded. The resulting sample was composed of 212 women ranging in age from 18 to $79(\mathrm{M}=31.59$; $\mathrm{SD}=12.79, n=212)$. The frequency of races in this sample was as follows: $53.2 \%$ identified as White, $12 \%$ identified as either AfricanAmerican, Hispanic/Latino, Asian/Pacific Islander, Middle Eastern, Native American or "Other," and $34.8 \%$ declined to indicate their race. The sample was well educated, with $49.2 \%$ reporting some higher education.

\subsection{Method}

Demographic questions included the following: "Are you in a romantic relationship?" "Do you live with your romantic partner?" "Do any of your children live with you?" "Do you live with one or more of your parents?” Given that these questions do not allow us to create a variable measuring living with male kin that is directly comparable to that employed in Study 1, to be conservative, we restrict Study 2 to re-testing predictions (i) and (ii).

Study 2 included the same dependent measure used in Study 1, a single item in which participants identified their level of concern on a 1-4 scale from "not at all worried" to "very worried" with regard to being the victim of sexual assault. Importantly, in addition to this single item, participants also completed the Fear of Rape Scale (FORS; SENN and DZINAS 1996), a standard, accepted measure of fear of rape having high construct validity and reliability. The FORS consists of 30 items such as "I am afraid of being sexually assaulted," "I carry objects (keys, knife something sharp) when I walk alone at night," and "I ask friends to walk me to my car/the subway if it is late at night;" participants were asked to respond on a scale anchored by $1=$ strongly disagree and $7=$ strongly agree, with $4=$ don't know/not applicable at the midpoint. The FORS yields a single factor; in our sample, $\alpha=.938$.

\subsection{Results}

We re-tested both predictions (i) and (ii) in a single one-way ANOVA model of a mean comparison of four groups - women not in a relationship $(\mathrm{M}=2.47$, $\mathrm{SD}=0.97, n=71$ ); women in a relationship but not living with their partner $(\mathrm{M}=2.58, \mathrm{SD}=1.06, n=33)$; women without children living with their romantic 
partner $(\mathrm{M}=2.72$, $\mathrm{SD}=0.87, n=58)$; and women living with their romantic partner and offspring $(\mathrm{M}=2.25, \mathrm{SD}=1.01, n=40)$ - with the single-item dependent measure, concern with sexual assault. This analysis revealed no significant difference between these groups $(p=.12)$. As in the case of Study 1 , we also computed post-hoc tests with Bonferroni correction and found that all individual mean comparisons between groups were nonsignificant ( $p$ 's >.11).

Next, we tested both predictions in the same manner using the FORS (including all items as a single factor) as the dependent measure in place of the single-item dependent variable. Again, the four groups - women not in a relationship ( $\mathrm{M}=4.11, \mathrm{SD}=1.07, n=73)$; women in a relationship but not living with their partner $(\mathrm{M}=4.08, \mathrm{SD}=1.19, n=36)$; women without children living with their romantic partner $(\mathrm{M}=4.20, \mathrm{SD}=0.98, n=57)$; and women living with their romantic partner and offspring $(\mathrm{M}=4.48, \mathrm{SD}=1.23, n=40)$ - did not significantly vary from each other $(p=.31)$. Again, we also computed post-hoc tests with Bonferroni correction and found that all individual mean comparisons between groups were nonsignificant ( $p$ 's >.50). Once again, testing the independence of age and cohabitation status (prediction [iv]) was not possible for either dependent measure, as there was no effect for cohabitation.

\subsection{Discussion}

Testing predictions (i) and (ii) with a second sample and a better dependent measure again failed to produce significant differences in fear of rape between women in pair-bonds and those not. These results are inconsistent with the prediction, derived directly from the RSH, that, driven by the putative extra cost of potential abandonment as a result of sexual assault faced by women in pair-bonds, natural selection has crafted psychological mechanisms that serve to calibrate rape-avoidance behaviors in light of relationship status.

\section{GENERAL DISCUSSION}

We question the validity of existing evidence (THORNHILL and THORNHILL 1990a, 1990b, 1990c; McKibBin, SHACKELFORD, Miner, BATES, and LidDle 2011) adduced in support of the hypothesis that married women's responses to sexual assault or the risk thereof reflect the added potential cost of a reduction in investment by their mate. As discussed above, the methods employed to collect said evidence are subject to multiple substantive problems. Our own attempts to verify the RSH consistently produced null results. Admittedly, our methods are subject to limitations, as measuring attitudinal fear of rape is an indirect avenue for testing predictions regarding the costs of rape; hence, in future studies, it would be preferable to further examine the consequences of rape. Some readers may conclude that a possible wrinkle in linking our attitudinal measures of fear of rape to the sequelae of rape is the fact that individuals can be poor at forecasting how they will feel after taxing 
events (for an overview of this literature, see GILBERT and EBERT 2002). However, while the imperfect nature of people's attempts to project themselves into the future could conceivably lead women to underestimate the costs of rape, in general, the literature addressing the former topic concerns the details of forecasters' accuracy, not their general ability to recognize events that are or are not in their best interests. At the pragmatic level, it is difficult to imagine that women would fail to recognize that being raped is a terrifying and traumatic experience. At the theoretical level, it would be a perverse design indeed were it the case that adaptations that shape behavior in light of the relative costs of sexual assault were only operational post hoc, i.e., after rape, when the costs have already been incurred, or are imminent. Surely, if such adaptations exist, they must be active prospectively, and, when successful, will generally preclude rape entirely.

Consonant with the above position, as noted earlier, an existing literature contradicts the notion that pair-bonded victims of rape experience poorer adjustment than victims not in a committed long-term sociosexual relationship. At the same time, we acknowledge that, for multiple reasons, there is a high risk of marital discord following sexual assault committed by a third party. We suspect that additional clarity in this regard may be achieved by examining the male partner as a contributing factor in such discord, particularly the extent of his proprietary attitude toward the victim, and his corresponding sense that his rights of exclusive sexual access have been violated (WILSON and DALY 1992), as well as his sense of failure stemming from his inability to shelter his partner from harm (MCCAHILL, MEYER, and FISCHMAN 1979). Additional variation in marital discord following sexual assault by a third party may stem from the woman's conception risk, including her use of contraception, her position in the menstrual cycle, and, perhaps, coital versus noncoital rape.

Relatedly, in our reading of the existing literature, there is no support for $\mathrm{T} \&$ $\mathrm{T}$ 's highly original notion that married women who have been beaten in the course of sexual assault experience significantly less psychological pain or less marital discord. Rather, we endorse a simpler logic in which the greater the woman is harmed, the more difficult her recovery, and, correspondingly, the greater the potential for marital discord stemming from the stresses that such recovery places on the relationship.

In light of the analytic problems and inconsistent or negative results in T \& T's four articles on the topic of the sequelae of rape, the lack of extensive support from other investigations, and our own uniformly null results in regard to the RSH, we suggest that it is time to discard oversimplifications that portray the results of the original investigations as conclusive. Rape is a deeply disturbing social ill and a profound tragedy for victims and those close to them. Neither social policy nor clinical practice are advanced by accepting as proven ideas that, howsoever logical and theoretically cogent they may be, nevertheless remain largely unsupported by empirical evidence. Rather, we suggest: that every effort should be made to 1) directly retest the RSH using sexual assault data that assesses the interaction between 
demographic variables and psychological sequelae; and 2) continue to indirectly test these predictions, as we and MCKIBBIN et al. (2011) have sought to do.

\section{ACKNOWLEDGEMENTS}

We thank Colin Holbrook and H. Clark Barrett and several anonymous reviewers for useful feedback on previous versions of this manuscript.

\section{Notes}

${ }^{1}$ While a number of prior critics frame their critiques in terms that make it clear that they have theoretical - or, worse yet, ideological - axes to grind, nonetheless, the substantive points raised are independent of the motives of said authors.

${ }^{2}$ While ideally we would also test the RSH's ancillary prediction that physical injuries will interact with marital status to mitigate the psychological pain of rape, as this cannot be examined using prospective attitudes, our evaluation of this claim must be limited to the observation that there is little compelling evidence, in T \& T 1990c or elsewhere, that it is true.

\section{REFERENCES}

BRÖDER, A., \& HOHMANN, N. (2003): Variations in risk-taking behavior over the menstrual cycle: An improved replication. Evolution and Human Behavior, 24, 391-398. doi: 10.1016/S1090-5138(03)00055-2

BRowNMILLER, SUSAN. 2000: "Rape on the brain (Review of A natural history of rape by Randy Thornhill and Craig T. Palmer).” Feminista! 3:9.

Chavanne, T. J., \& GalluP, G. G. (1998): Variation in risk taking behavior among female college students as a function of the menstrual cycle. Evolution and Human Behavior, 19, 27-32. doi: 10.1016/S1090-5138(98)00016-6

Coyne, Jerry A. 2000: "Of vice and men (Review of Thornhill and Palmer's A natural history of rape).” New Republic, April 3, pp. 27-34.

Coyne, Jerry A. and ANDrew BerRy. 2000: "Rape as an adaptation (Review of Thornhill and Palmer's A natural history of rape).” Nature, 404:121-22.

ELLIS, B. J. (1992): The evolution of sexual attraction: Evaluative mechanisms in women. In: J. H. BARKow, L. COSMIDES, \& J. TOOBY. (Eds.), The adapted mind: Evolutionary psychology and the generation of culture (pp. 267-288). New York: Oxford University Press.

Fleischman, D. S., Perilloux, C. \& Buss, D. M. (2012): Avoidance of contexts of sexual assault across the menstrual cycle. Manuscript submitted for publication.

FrEesE, J. (2000): What should sociology do about Darwin? Evaluating some potential Contributions of sociobiology and evolutionary psychology to sociobiology. Dissertation, Sociology, Indiana University.

Garver-Apgar, C. E., GAngestad, S. W., \& Simpson, J. A. (2007): Women's perceptions of men's sexual coerciveness change across the menstrual cycle. Acta Psychologica Sinica, 39, 536-540.

GILBERT, D. \& EBERT, J. E. J. (2002): Decisions and revisions: The affective forecasting of changeable outcomes. Journal of Personality and Social Psychology, 82, 503-514.

JonES, O. D. (1999). Sex, culture, and the biology of rape: Toward explanation and prevention. California Law Review, 87, 827-941. 
KilPATRICK, D. G., Veronen, L. J., \& BeSt, C. L. (1985): Factors predicting psychological distress among rape victims. In Charles R. Figley (Ed.) Trauma and its wake: Volume I: The study and treatment of post-traumatic stress disorder. Bristol, PA: Brunner/Mazel.

Koss, M. P. (2000): "Evolutionary models of why men rape: Acknowledging the complexities." Trauma, Violence, and Abuse 1:182-190.

Koss, M. P., Dinero, T. E., Seibel, C. A. \& Cox, S. L. (1988): Stranger and acquaintance rape: Are there differences in the victim's experience? Psychology of Women Quarterly, 12, $1-24$.

McCahill, T. W., Meyer, L. C., \& Fischman, A. M. (1979): The aftermath of rape. Lexington, MA: Lexington Books.

McKibbin W.F., Shackelford, T.K., Goetz, A.T., Bates, V.M., StarRatt, V.G. (2009): Development and initial psychometric assessment of the rape avoidance inventory. Personality and Individual Differences, 46, 336-340.

MCKibBin W.F., SHACKELFORD, T.K., MinER, E.J., BATES, V.M., LidDLE, J.R. (2011): Individual differences in women's rape avoidance behaviors. Archives of Sexual Behavior, 40, 343-349.

Mesnick, S. L. (1997): Sexual Alliances: Evidence and evolutionary implications. In P. A. Gowaty (Ed.) Feminism and Evolutionary biology: Boundaries, intersections and frontiers (pp. 207-260). New York, Chapman \& Hall.

MoRgAN, J.B. (1981): Relationship between rape and physical damage during rape and phase of sexual cycle during which rape occurred, Unpublished Ph.D. dissertation, Department of Educational Psychology, University of Texas at Austin.

Resick, P. A., Calhoun, K. S., AtKeson, B. M., and Ellis, E. M. (1981): Social adjustment in victims of sexual assault. Journal of Consulting and Clinical Psychology, 49, 705-712.

REsick, P. A. (1993): The psychological impact of rape. Journal of Interpersonal Violence, 8, $223-255$.

Rogel, M.J. (1976): Biosocial aspects of rape, Unpublished Ph.D. dissertation, Department of Psychology, University of Chicago.

Saunders, B. E., Kilpatrick, D. G., Hanson, R. F., Resnick, H. S. \& Walker, M. E. (1999): Prevalence, case characteristics, and long-term psychological correlates of child rape among women: A national survey. Child Maltreatment, 4, 187-200.

Senn, C. Y. \& Dzinas, K. (1996). Measuring fear of rape: A new scale. Canadian Journal of Behavioral Science, 28, 141-144.

SHAFfer, J. P. (1995): Multiple hypothesis testing. Annual Review of Psychology, 46, 451-484.

Simkin, M. V. \& RoychowdhURY, V. P. (2003). Read before you cite! Complex Systems, 14, 269-274.

Snyder, J. K. \& Fessler, D. M. T. (2013): Reexamining individual differences in women’s rape avoidance behaviors. Archives of Sexual Behavior, 42, DOI 10.1007/s10508-012-9987-6

Snyder, J. K., Fessler, D. M. T., Tiokhin, L., Frederick, D., Lee, S. S., \& Navarrete, C. D. (2011): Trade-offs in a dangerous world: Women's fear of crime predicts preferences for aggressive and formidable mates. Evolution and Human Behavior, 32, 127-137.

The Crime Reduction Centre (2000, July): British fear of local crime survey. Retrieved May 1, 2005, from Crime Reduction Toolkits onlineviahttp://www.crimereduction.gov.uk/toolkits/fc0401.htm.

ThornhiLl, N. W. (1996): Psychological adaptation to sexual coercion in victims and offenders. In D. M. Buss \& N. Malamuth (Eds.), Sex, power, conflict (pp. 90-104). New York: Oxford University Press.

THORNhill, N. W. \& THORNhiLl, R. (1990a): An evolutionary analysis of psychological pain following rape: I. The effects of victims age and marital status. Ethology and Sociobiology, 11, 155-176. 
ThORnhill, N. W. \& ThORNhill, R. (1990b): An evolutionary analysis of psychological pain following rape: II. The effects of stranger, friend, and family member offenders. Ethology and Sociobiology, 11, 177-193.

THORNHILl, N. W. \& THORNHILL, R. (1990c): An evolutionary analysis of psychological pain following rape. III: Effects of force and violence. Aggressive Behavior, 16, 297-320.

Thornhill, N. W. \& THORNHILl, R. (1991): An evolutionary analysis of psychological pain following human (Homo sapiens) rape: IV. The effect of the nature of the sexual assault. Journal of Comparative Psychology, 105, 243-252.

Thornhill, R. \& PALmer, C. T. (2000): A natural history of rape: Biological bases of sexual coercion. Cambridge, MA: MIT Press.

Wilson, M. \& MesnicK, S. L. (1997): An empirical test of the bodyguard hypothesis. In Patricia Adair Gowaty (Ed.) Feminism and evolutionary biology: Boundaries, intersections and frontiers (pp. 505-511). New York, Chapman \& Hall.

Wilson, M. \& DALY, M. (1992):The man who mistook his wife for chattel. In J.H. Barkow, L. Cosmides, \& J. Tooby (Eds.), The adapted mind: Evolutionary psychology and the generation of culture (pp. 289-322). New York: Oxford University Press. 